\title{
Numerical Thermo-Mechanical Modelling of Stress Fields and Residual Constraints in Metallic Targets Subject to Laser Shock Processing
}

\author{
M. Morales, C. Molpeceres, J.A. Porro, A. García-Beltrán, J.L. Ocaña \\ Centro Láser UPM. Universidad Politécnica de Madrid. \\ Campus Sur UPM. Edificio La Arboleda. Ctra. de Valencia, km. 7,300. 28031 Madrid. SPAIN. \\ Tel.: (+34) 913363099. Fax: (+34) 913365534. email: miguel.morales@upm.es,
}

Keywords: Laser shock processing, residual stresses, surface properties, mechanical effects, thermal effects, numerical simulation.

\begin{abstract}
.
In the analysis of the thermomechanical behaviour of the target material subject to Laser Shock Processing (LSP), most of the simplified models used for the analysis of its residual shocked state rely on rather simple estimations or material response equations that rarely take into account a detailed description of the material subject to a simultaneous dynamic compression and either deformation-induced or plasma-driven thermal heating.

The calculational system developed by the authors (SHOCKLAS) includes a coupled analysis of the pressure wave applied to the target material as a result of the plasma buildup following laser interaction and the shock wave propagation into the solid material with specific consideration of the material response to thermal and mechanical alterations induced by the propagating wave itself (i.e. effects as elastic-plastic deformation, changes in elastic constants, etc.). The model is applicable to the typical behaviour shown by the different materials through their dynamic strain-stress relations.

In the present paper, the key features and several typical results of the developed SHOCKLAS calculational system are presented. In particular, the application of the model to the realistic simulation (full 3D dependence, non linear material behaviour, thermal and mechanical effects, treatment over extended surfaces) of LSP treatments in the experimental conditions of the irradiation facility used by the authors is presented
\end{abstract}

\section{Introduction}

Laser shock processing is being considered as a competitive alternative technology to classical treatments for improving fatigue, corrosion cracking and wear resistance of metallic materials, and, on the basis of the commercial availability of new powerful laser sources able to provide intensities exceeding the $\mathrm{GW} / \mathrm{cm}^{2}$ level $[1,2]$.

However, although significant work from the experimental side has contributed to explore the optimum conditions of application of the treatments, only limited attempts have been developed in the way of full comprehension and predictive assessment of the characteristic physical processes. Additionally, some relevant work has been made in the line of prediction and characterization of the mechanical properties enhancement of material treated by the LSP technique.

A fundamental reason for the referred lack of predictive capability of LSP processes is their inherent physical complexity, specially stemming on the coexistence of different material phases (including plasma) developing and interacting under the action of the high intensity laser beam.

\section{Physical Basis of LSP Processes}

Laser Shock Processing (LSP) is based on the application of a high intensity pulsed Laser beam (I $>1 \mathrm{GW} / \mathrm{cm}^{2} ; \tau<50 \mathrm{~ns}$ ) at the interface between the metallic target and the surrounding medium (a transparent confining material, normally water) forcing a sudden vaporization of the metallic surface into a high temperature and density plasma that immediately develops inducing a shock 
wave propagating into the material (see Fig. 1a). The shock wave induces plastic deformation and a residual stress distribution in the target material (if its peak pressure is greater than the dynamic yield limit) (see Fig. 1b).

The material is also heated by the thermal flux generated upon laser material interaction and by the plastic deformation work. This produces a deleterious effect due thermal stress relaxation and tensile stresses generation in a narrow layer under the target free surface [3].

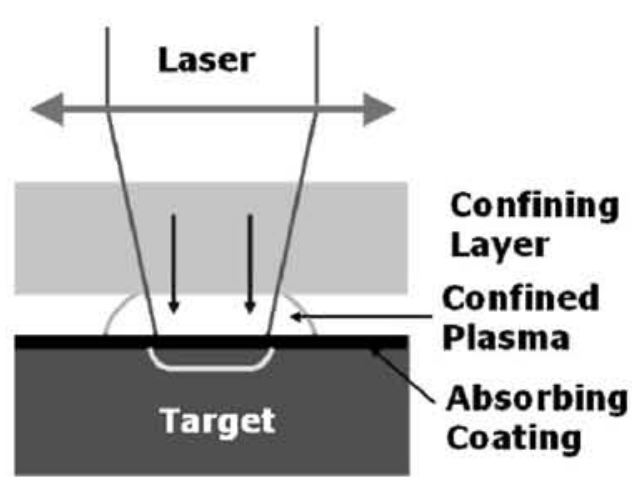

(a)
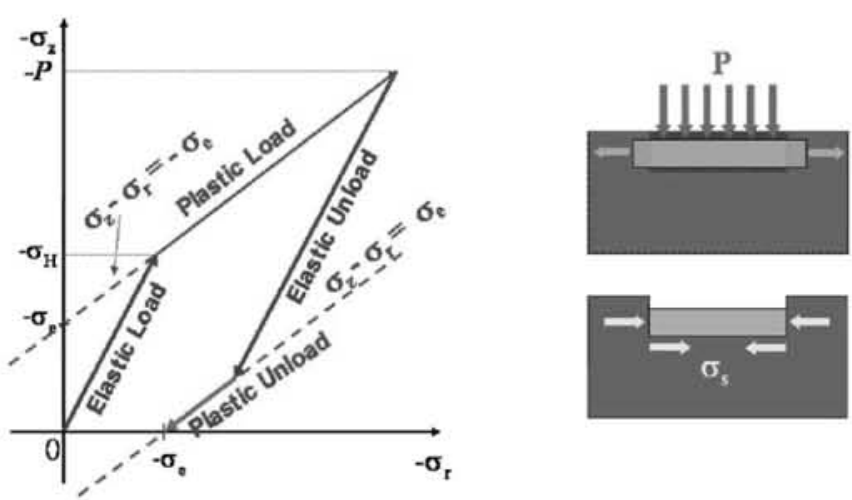

(b)

Figure 1: Schematic representation: (a) LSP process. (b) Plastic deformation and residual stresses induction.

\section{Numerical modeling of LSP processes}

Provided the large amount of physical phenomena arising in the considered processes, the corresponding modelling, including the formation of a vapour/plasma phase, the generally far from equilibrium ionization-recombination processesin this plasma, its thermofluiddynamic behaviour under extreme pressure and temperature conditions (typically leading to pressure/shock waves), etc., requires a deep understanding of the physics underlying their appearance, and appears as absolutely needed for the reliable predictive assessment of the material evolution under irradiation.

The most popular methods reported in the literature for the analysis of the LSP phenomenology $[2,5-6]$ try to induce the intensity and temporal profile of the shock wave launched into the treated solid material by means of the analysis of the impulse conservation between the external interface of such material and the frontier of the confining material without any reference to the detailed physics of the plasma formation process taking place in the outermost layers of the solid target: this plasma is assumed to be built up to certain degree as a consequence of the initial laser energy deposition, but no analysis is provided about its real dynamics.

From the point of view of the acceleration of the confining medium as a consequence of the excess pressure due to the expansion of the generated plasma, the simplified models proposed in the cited references can provide adequate results that can even be experimentally contrasted. However, from the point of view of the actual compression dynamics of the solid target (the main objective of the study and for which the contrast to experimental results is far more complicated), such simplified models are presumably not able to provide a correct estimate of the pressure/shock waves effectively launched, at least in the initial moments of the laser interaction, in which the complex physics related to plasma ionization dynamics can substantially modify the target state in view of the subsequent process development.

The appropriate description of the LSP process requires a three level description providing the adequate interconnection of the data obtained in each phase in a self-consistent way from the physical point of view.

The referred three-level description includes: 
i) Analysis of the plasma electronic population dynamics, including consideration of breakdown phenomenology in dielectric media,

ii) Simulation of the hydrodynamic phenomenology arising from plasma expansion between the confinement layer and the base material

iii) Analysis of the propagation and induction of permanent structural changes by shock wave evolution in bulk material

A simulation model (SHOCKLAS), dealing with the main aspects of LSP modelling in a coupled way, has been developed by the authors $[5,6]$.

\section{Laser Plasma Interaction}

The problem of laser-plasma interaction at very high intensities has been a subject of permanent interest from the appearance of the first lasers [7,8]. HELIOS is a 1-D radiation-hydrodynamics code that is used to simulate the dynamic evolution of laser created plasmas [9].

Fluid methods describe the dynamics of a continuous medium and are thus applicable when the mean free path of the constituent particles is small compared to the characteristic dimensions within the system. In this kind of problems, the fluid is generally described by its thermodynamic state and by the velocity of flow. The state variables are related by the material equation of state (E.O.S.). The well-known Navier-Stokes equations of fluid mechanics yield five relations expressing the basic conservation laws of physics applied to the moving fluid [10].

Hydrodynamic codes for the analysis of laser matter interaction at high intensities generally use a one (common for ions and electrons) or two (differentiated for ions and electrons due to the weak energy coupling between the two populations) temperature-fluid scheme. Electrons and ions are assumed to flow as one fluid what implies no charge separation.

Radiation energy transport can be included as either a third temperature equation (assuming a local Planck distribution), o by means of an energy dependent treatment of the photon distribution function. In HELIOS opacities are based on tabulated multi-group PROPACEOS data [9] radiation emission and absorption terms are coupled to the electron temperature equation. Multi-frequency radiation intensities are computed using either a flux limited radiation diffusion model, or a multiangle model based on the method of short characteristics.

From the mathematical point of view, the equations describing the plasma fluid-dynamic motion are hyperbolic and have solutions with a characteristic propagation speed, admitting, in absence of dissipative terms, discontinuous solutions than can create difficulties for finite difference schemes. The introduction of the Von Neumann artificial viscosity effectively smoothes the shock [10].

Material EOS properties are fundamental in hydrodynamic codes. HELIOS EOS are based on either SESAME tables [11] or PROPACEOS [9] tables.

Laser energy deposition is computed using an inverse Bremsstrahlung model, with the restriction that no energy in the beam passes beyond the critical surface.

\section{Target thermo-mechanical behavior}

Coupled treatment of thermal and mechanical transient processes characteristic of LSP is needed for a realistic characterization of material surface properties modification directily suitable for comparison to experimental tests. On the basis of the time-dependent pressure and heat flux profile calculated by HELIOS, HARDSHOCK solves the shock propagation problem into the solid material, with specific consideration of the material response to thermal and mechanical alterations induced by the propagating wave itself (i.e. effects as elastic-plastic behaviour, changes in elastic constants, phase changes, etc.). The target material subject to LSP is heated due to two main mechanisms: direct laser interaction heating (input from HELIOS simulations) and heating by plastic deformation work. The resulting temperature rise is correspondingly computed, so that this temperature change produces, in turn, a local thermal expansion of the target material whose subsequent thermal strains have to be consistently calculated [3]. 
For this kind of problems, a 3D version, based in the FEM commercial code ABAQUS® is used. From the point of view of time differencing, the usual strategy of explicit differencing for the initial fast shock propagation phase followed by standard implicit differencing for the analysis of the final residual stresses equilibrium is not used, instead only explicit differencing has been used with long time evolutions in order to reach thermal and stress equilibration. The FEM element used for the mechanical simulation is an 4-node brick reduced integration with hour glass control bilineal, namely CAX4RT, for axissymmetric studies of the effect of one single pulse and an 8-node brick reduced integration with hourglass control trilinear, namely C3D8RT, for fully 3D simulations with geometric overlapping of pulses.

\section{Model results}

\section{Study of LSP produced plasma}

HELIOS code has been applied to the simulation of plasma dynamics of an aluminium target subject to LSP conditions $\left(\lambda=1064 \mathrm{~nm}, \mathrm{~F}=84 \mathrm{~J} / \mathrm{cm} 2\right.$ and $\left.\tau_{\mathrm{FWHM}}=9 \mathrm{~ns}\right)$ but with confining layer variations (medium and thickness). Results for space and time dependent variation of electron temperature (Fig. 2a) and ion density (Fig. 2b) for a Water/Al plasma produced in LSP conditions are shown. Plasma (high temperature region) is close to the surface (see Fig. 2a) while shock wave (high density region) is moving very fast (see Fig. 2b).
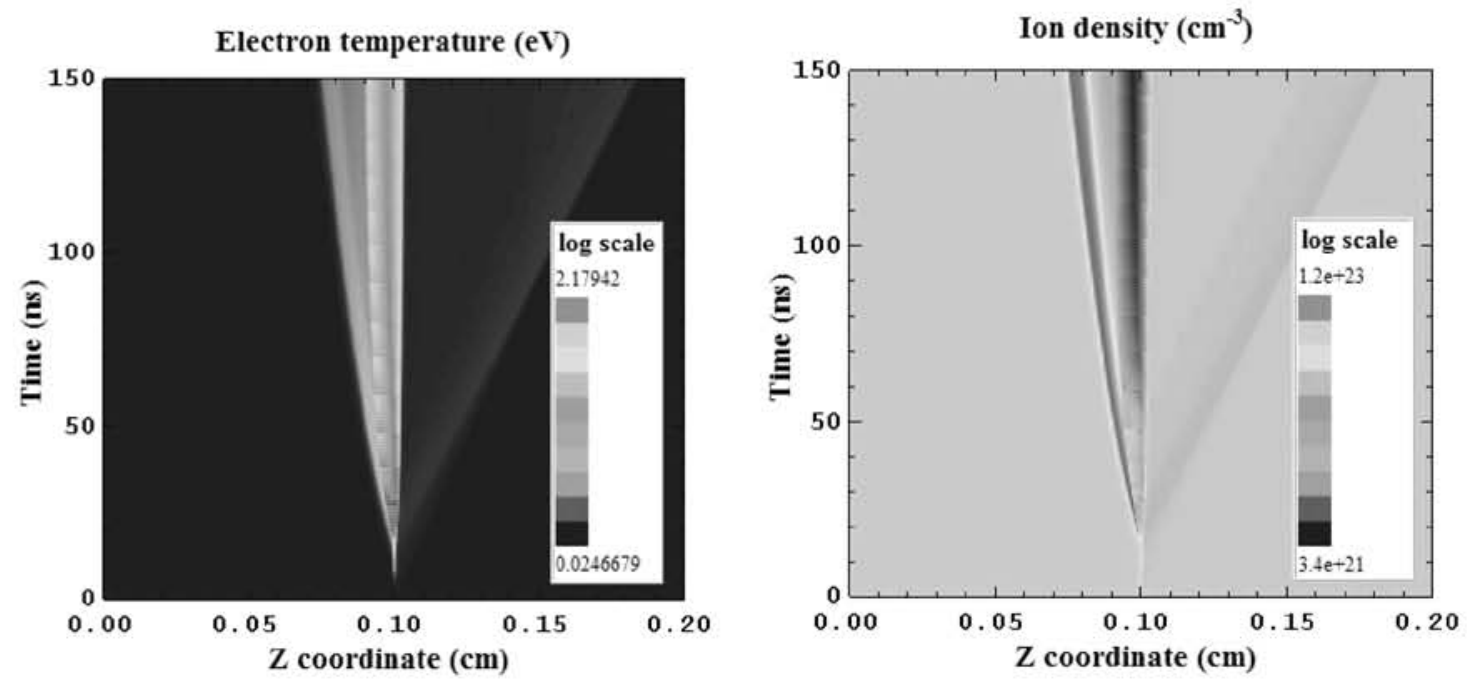

Figure 2: Water/Al plasma produced in LSP conditions:

(a) Space-time variation of pressure. (b) Space-time variation of ion density.

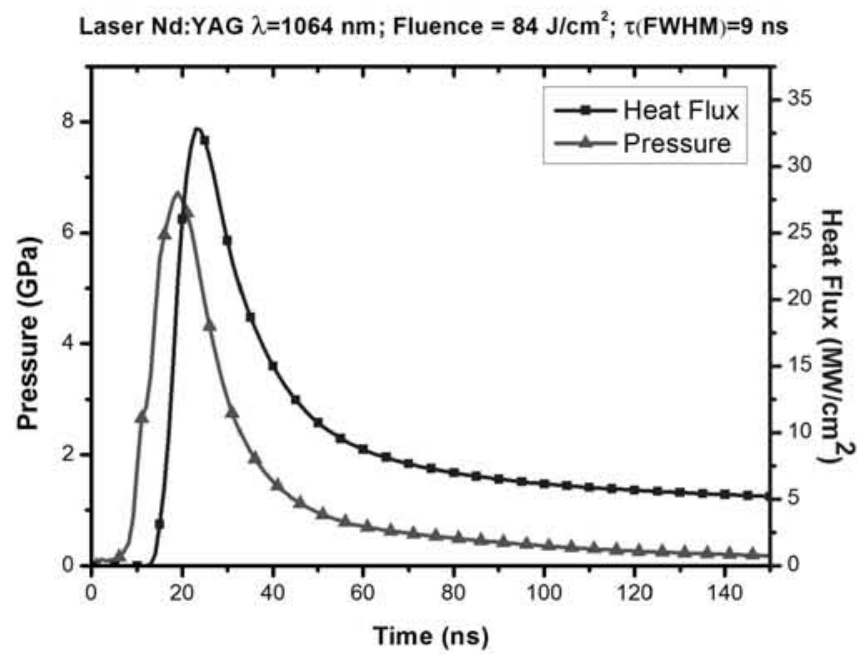

Figure 3: Plasma pressure and heating rate for a Water/Al plasma produced in LSP conditions. 
In Fig. 3 plasma pressure and heating rate obtained for a Water/Al plasma produced in LSP conditions are displayed. These are the boundary conditions that are applied in the surface of the target in the FEM code for the determination of the residual stress field.

\section{Influence of pressure pulse and heat flux on the residual stress distribution}

The analysis of the residual stresses induced by a single shot with full consideration of thermal and mechanical effects has been performed, both considering individually either mechanism and in a fully coupled way. Laser spot diameter is $1.5 \mathrm{~mm}$. The corresponding residual stress distribution is represented in Fig. 4.
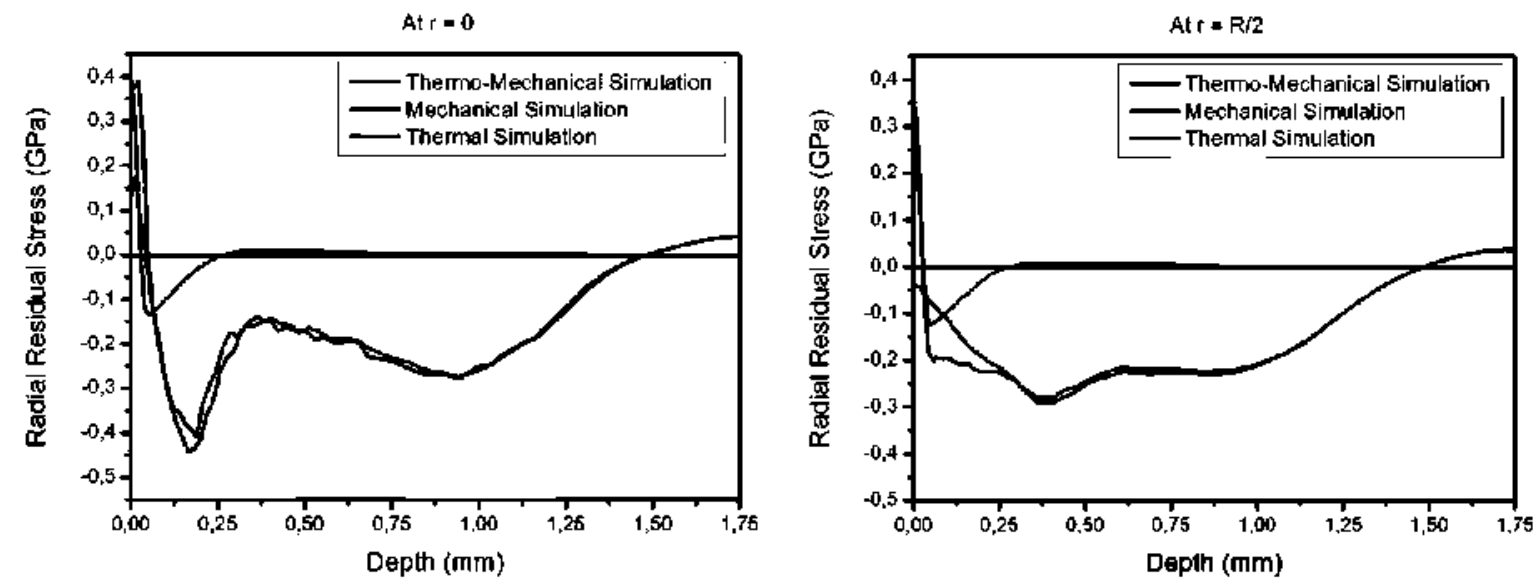

Figure 4: Radial residual stress in a thermal, mechanical or fully coupled simulation:

(a) Axis at spot center. (b) Space-time variation of ion density.

In this figure, the opposite effects of the thermal wave and the mechanical shock wave on the material residual stress fields near the free surface are clearly shown: while the pure consideration of the mechanical effects induced by the shock wave launched into the material by the laser generated plasma results in an effective target compression until rather important values (for a single laser pulse) of compressive residual stresses, the effect of the thermal flux entering the piece as a direct consequence of contact and radiation from such plasma has clearly a deleterious effect over such residual stresses field near the surface, but the stress after this first layer is increased due to stress self equilibration.

\section{Evaluation of the residual stress obtained by application of adjacent pulses}

In this case the overlapping of pulses (in the same conditions previously discussed) has been considered. The corresponding residual stress distribution is represented in Fig. 5.
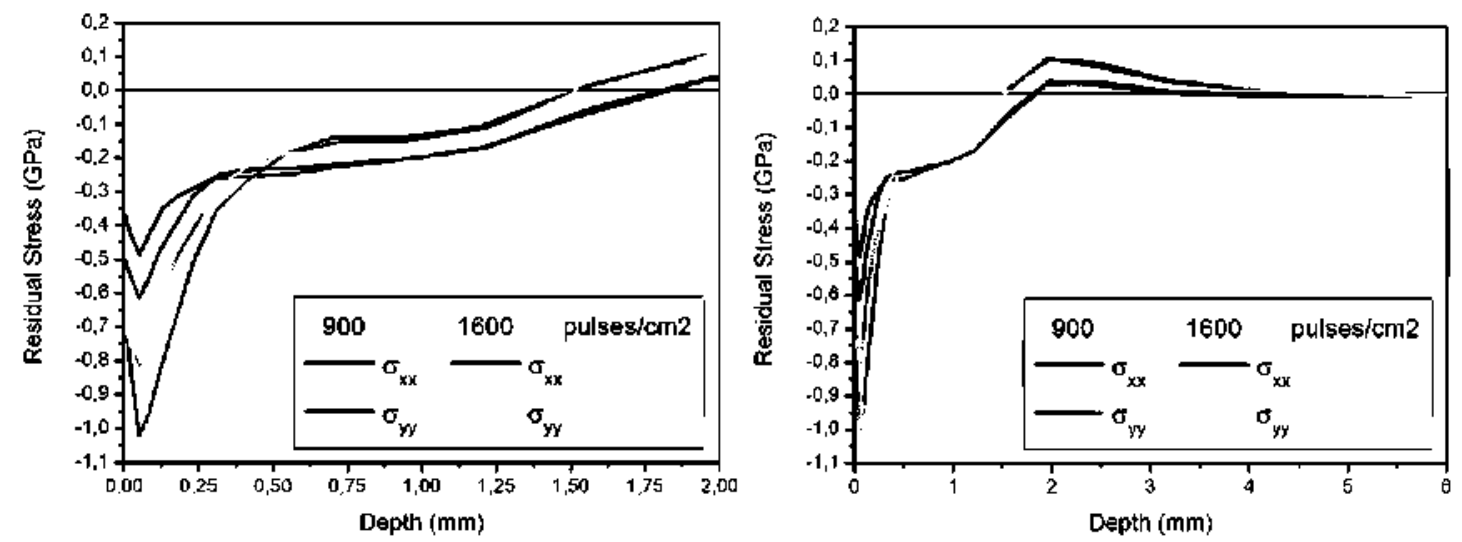

Figure 5: Residual stress obtained with overlapping of pulses:

(a) Near the surface. (b) Full thickness of the sample. 
The effect of several pulses in the same point overcomes the tensile stress and produces compression in the surface. There is an increase in the residual stress close to the surface due to self equilibration and after that it starts to decrease until a new tensile region starts due to self equilibration.

\section{Discussion}

The phenomenology involved in LSP processes is complicated mostly because of their characteristic laser-plasma interaction dynamics and coupled thermo-mechanical target behaviour. The need of including a detailed plasma code and a fully coupled thermo-mechanical target behavior in any numerical system for the predictive assessment of LSP treatment has been shown.

In this line, the authors have developed a system of coupled computational modules dealing with the different physical evolution regimes present in the process.

The results obtained show the need of taking into account thermal effects and that tensile stresses produced in laser shock processing without coating are overcome with laser spot overlapping.

\section{Acknowledgments}

Work partly supported by MICINN/MEC (Spain; Projects DPI2005-09152-C02-01 and MAT200802704/MAT) and EADS-Spain.

\section{References}

[1] Y. Sano, N. Mukai, K. Okazaki and M. Obata, Nuclear Instruments \& Methods in Physics Research, Section B Vol. 121 (1997), p. 432

[2] J.L. Ocaña, C. Molpeceres, J.A. Porro, G. Gómez and M. Morales, Appl. Surf. Sci, Vol. 238 (2004), p. 501

[3] J.L. Ocaña, M. Morales, C. Molpeceres, J.A. Porro and A. García-Beltrán, Mater. Sci. Forum Vol. 539-543 (2007), p. 1116

[4] L. Berthe, R. Fabbro, P. Peyre, L. Tollier and E. Bartnicki, J. Appl. Phys. Vol. 82 (1997), p. 2826

[5] J.L. Ocaña, M. Morales, C. Molpeceres and J. Torres, Appl. Surf. Sci. Vol. 238 (2004), p. 242

[6] M. Morales, J.L. Ocaña, C. Molpeceres, J.A. Porro and A. García-Beltrán, Surface \& Coatings Technology Vol. 202 (2008), p. 2257

[7] F. Cottet and J.P. Romain, Phys. Rev. A Vol. 25 (1982), p. 576

[8] R.J. Trainor and Y.T. Lee, Phys. Fluids Vol. 25 (1982), p. 1898

[9] J. J. MacFarlane, I. E. Golovkin, and P. R. Woodruff, J. Quant. Spectrosc. Radiat. Transfer Vol. $99(2006)$, p. 381

[10] J.L. Ocaña, Lasers in Engineering Vol. 3 (1994), p. 301

[11] S.P. Lyon and J.D. Johnson: SESAME: The Los Alamos National Laboratory Equation of State Database. Technical report, LA-UR-92-34 (Los Alamos National Laboratory, Los Alamos 1992) 